\title{
LA MÉDECINE PRÉVENTIVE DU CHIEN ET DU CHAT : HISTOIRE ET STRUCTURATION D'UNE DISCIPLINE CLINIQUE
}

\author{
PREVENTIVE MEDICINE FOR CATS AND DOGS: HISTORY AND \\ STRUCTURING OF A CLINICAL DISCIPLINE
}

Par Ludovic FREYBURGER ${ }^{1}$

(Communication présentée le 4 février 2021, manuscrit accepté le 18 juin 2021)

\begin{abstract}
RÉSUMÉ
La médecine préventive est une discipline clinique des Sciences Vétérinaire qui s'attache tout au long de la vie de l'animal, à préserver son état de bonne santé, à identifier et limiter les risques de sa dégradation, ainsi qu'à empêcher la transmission de zoonoses. Cette définition a été établie en 2016 par les enseignants de médecine préventive des quatre écoles vétérinaires françaises et répond à une nécessité de structurer une discipline clinique qui représente un des piliers historiques de la profession vétérinaire, et qui répond à une forte demande de repères fiables et rigoureux des propriétaires dans une société en constante évolution. Nouvelle approche, nouvelle méthodologie, nouveau langage, nouvelle communication, nouveau rapport avec le propriétaire de chien et de chat. II s'agit donc d'un nouveau paradigme pour une pratique quotidienne de la médecine vétérinaire au service du maintien de la bonne relation Homme-Animal.
\end{abstract}

Mots-clés : médecine préventive, vaccination, chien, chat, consultation, enseignement, relation homme-animal.

\begin{abstract}
-ABSTRACT
Preventive medicine is a clinical discipline of Veterinary Science that focuses throughout the life of the animal, to preserve its good health, to identify and limit the risks of its degradation, as well as to prevent the transmission of zoonoses. This definition was established in 2016 by the preventive medicine teachers of the four French veterinary schools and responds to a need to structure a clinical discipline that represents one of the historical pillars of the veterinary profession, and which responds to a strong demand for reliable and rigorous benchmarks of owners in an ever-changing society. New approach, new methodology, new language, new communication, new relationship with dog and cat owner. It is therefore a new paradigm for a daily practice of veterinary medicine in the service of maintaining the right human-animal relationship.

Key-Words: preventive medicine, vaccination, dog, cat, consultation, teaching, human-animal relationship
\end{abstract}

\section{INTRODUCTION}

La pratique vétérinaire évolue et se structure fortement depuis plusieurs années : l'apparition des spécialités et l'évolution de la réglementation a permis de proposer aux propriétaires d'animaux une offre de soin de plus en plus pointue et perfectionnée, qui répond à la fois à leurs attentes, ainsi qu'à la vocation que tout vétérinaire embrasse lors son choix professionnel : soigner des animaux malades. En parallèle, l'animal dans la société est passé de la place d'animal utile et de production au statut d'animal de compagnie, pour maintenant être décrit par les propriétaires de chien et de chat comme étant un membre de la famille (Cohen, 2002). Les attentes des propriétaires ont donc fortement évolué, comme en témoignent les évolutions législatives sur le statut de l'animal, passé par la loi du 16 février 2015 de la condition de "bien meuble ", à celui d'être vivant doué de sensibilité " (Légifrance, Loi n²015-177, 2016) et la proposition de loi $\mathrm{n}^{\circ} 3661$ en cours d'étude et portée, entre autres, par le député Loïc Dombreval, vétérinaire de formation (Légifrance, Proposition de loi n³661, 2020). Ainsi, les attentes des propriétaires sur la prise en charge de la santé de leur animal progressent fortement, et le statut de soignant du vétérinaire

1 - DMV, PhD. Maître de Conférences en immunologie clinique (en disponibilité) - VetAgro Sup, Directeur de la Formation Vétérinaire - La Compagnie des Animaux / SantéVet, Président du Groupe d'Etude en Médecine Préventive - AFVAC, consultant en médecine préventive - VetAgro Sup. 35, rue de Marseille 69007 Lyon. Courriel : ludovic.freyburger@veterinaire.fr 
praticien évolue vers un statut de partenaire de la santé du chien et du chat. La médecine préventive occupe ainsi une place considérable dans cette évolution, en tant que discipline clinique nouvellement reconnue par la profession, bien que faisant partie du quotidien du praticien, ne serait-ce que par le biais de la vaccination dont l'histoire renseigne fortement sur les étapes du développement et de la structuration de la médecine préventive.

\section{ÉMERGENCE DE LA DISCIPLINE MÉDECINE PRÉVENTIVE : APPORTS DE LA VACCINA- TION ET DE SON HISTOIRE}

\section{Historique du développement de la vaccination}

Le 9 août 1721 eut lieu à la prison de Newgate, en Angleterre, une expérimentation visant à démontrer les bienfaits d'une technique que Lady Mary Worstley Montagu rapporta de son voyage accompagnant la mission diplomatique de son mari auprès de l'Empire ottoman à Constantinople. Six prisonniers, trois femmes et trois hommes condamnés à mort, furent publiquement variolisés par le docteur Maitland, chirurgien de son état, et peu coopératif sur le moment (Bazin, 2008). Les six condamnés survécurent et ne développèrent pas la variole. Fort du succès de cette opération, et avant de pratiquer l'acte sur les enfants royaux, il fut décidé - avec succès - de réitérer l'expérience sur cinq orphelins de Saint James à Westminster. Quelques années auparavant, de 1712 à 1714, une épidémie de peste bovine décimait jusqu'à 90\% du cheptel européen. Le 4 août 1761, Claude Bourgelat reçut l'autorisation royale de créer une École vétérinaire, « ... où l'on enseignera publiquement les principes et la méthode de guérir les maladies des bestiaux, ce qui procurera insensiblement à l'agriculture du Royaume les moyens de pourvoir à la conservation du bétail dans les lieux où cette épidémie désole les campagnes... ». L'histoire montrera que la vaccination contre cette maladie permettra finalement son éradication. Bien que les enfants royaux aient été ensuite variolisés, cette technique peina à s'imposer comme une pratique nécessaire pour lutter contre la variole, maladie contagieuse s'il en est et indépendante du statut social des malades. Edouard Jenner, médecin de campagne qui pratiquait la variolisation, avait observé que les vachers qui avaient contracté le cow-pox - la vaccine ou picote - restaient insensibles à la variolisation et étaient protégés contre la maladie (Genetet, 2000). Le 14 mai 1796, il inocula à James Phipps, enfant de huit ans, dépourvu d'antécédent variolique, la lymphe d'une pustule de cow-pox prélevée chez Sarah Nelmes. Il s'agissait du premier vaccin hétérologue, qui permit de protéger le jeune James Phipps du virus de la variole à partir du virus de la vaccine, un virus bovin proche du poxvirus de la variole humaine. Presqu'un siècle plus tard, en conclusion de son intervention au Congrès International de Médecine en août 1881, Louis Pasteur rendit hommage à Edouard Jenner en ces mots : "J'ai prêté, à l'expression de "vaccination ", une extension que la Science, je l'espère, consacrera comme un hommage au mérite et aux immenses services rendus par un des plus grands hommes de l'Angleterre, Jenner » (Pasteur, «EEuvres complètes »1933). La vaccination reste à ce jour un terme qui consacre par excellence le lien entre santé animale et santé humaine ayant permis d'éradiquer deux maladies, la première humaine en 1977 : la variole (officiellement reconnue comme éradiquée par l'organisation mondiale de la santé (OMS) le 9 décembre 1979) et la seconde animale : la peste bovine officiellement déclarée éradiquée par l'Office International des Epizooties (OIE), lors de sa $79^{\text {ème }}$ session générale en 2011 (OIE, Résolution $n^{\circ} 18$ : Déclaration de l'éradication mondiale de la peste bovine et mise en œuvre des mesures de suivi visant à maintenir l'absence de cette maladie dans le monde, 2011). La vaccination a été la première démarche médicale moderne de prévention. A partir d'un animal en bonne santé, l'application d'une stratégie médicale permet de conserver cette bonne santé. Pourtant, cette même démarche conserve un caractère particulier en ce sens, que, pour nombre des maladies infectieuses contre lesquelles il est possible de vacciner, la vaccination reste la seule et unique stratégie de lutte, et de loin la plus efficace. De plus, la particularité de la vaccination repose sur la lutte à priori contre une hypothétique infection, dont les conséquences pourraient être importantes et pour laquelle les traitements sont souvent inexistants. Cette infection n'est toutefois pas certaine et ses conséquences sont potentiellement inconnues pour le public consécutivement à l'alliance entre l'efficacité de la stratégie vaccinale et l'absence d'informations et de connaissances sur la maladie devenue plus rare (Soubeyran, 2003).

\section{Communication et information, des étapes clés dans le développement de la vaccination}

Pratique scientifique pourtant reconnue, la vaccination est remise en cause dans la société par une partie de la population (Larson, 2016). Si l'influence des professionnels de santé, entre autres, semble être primordiale pour établir un état de confiance envers la vaccination (Couteille-Brillet, 2019), cette opposition n'est pas nouvelle, et est apparue dès sa généralisation. L'analyse historique du développement de la vaccination montre en effet que les pratiques et les prises de positions actuelles ne sont pas d'origine récente.

Lors du développement de la variolisation, des mouvements populaires, religieux, philosophiques se sont opposés ardemment à la mise en place de cette pratique (Moulin, 1996). Une ségrégation a même été observée dans des milieux sociaux variés entre partisans et détracteurs de la technique, que ce soit au cours de l'expédition Balmis, qui dès 1803 a permis son déploiement d'Europe en Amérique notamment par le biais des colonies espagnoles au frais de la couronne royale, ou en Europe, comme Voltaire le souligne dans sa onzième lettre philosophique. Dans ce plaidoyer en faveur de la technique, il met en avant l'opposition entre européen continentaux et anglais sur de la prise en charge de la petite vérole, qui ne s'arrêtait pourtant ni aux frontières, ni aux barrières sociales. Lorsque les pratiques de vaccination pasteurienne ont été développées, Louis Pasteur usa de son influence dans les milieux médicaux et politiques pour communiquer et diffuser des informations scientifiques, parfois à demi-mot sur les procédés exacts. Ainsi, qu'il s'agisse du vaccin contre le choléra des poules, ou de façon encore plus démonstrative sur la 
vaccination contre le charbon, avec l'expérience publique menée à Pouilly-le-Fort, Louis Pasteur permit de montrer les bienfaits de la technique et il communiqua résolument à leur propos. Quelques années plus tard, dans le contexte de relations franco-allemandes tendues, il fit la démonstration de l'efficacité de la vaccination humaine contre la rage, et là encore médiatisa cette approche (Genetet, 2000 ; Bazin, 2008). La vaccination entra petit à petit dans les pratiques médicales, tant vétérinaire qu'humaine, et permit la prise en charge de beaucoup de maladies infectieuses. Bien qu'elles n'aient pas totalement disparu du paysage, ces maladies sont tellement moins présentes et leurs conséquences sont pour certaines tellement méconnues dans la vie quotidienne que nombre de personnes refusent de se faire vacciner, particulièrement en France (Raude, 2016). L'hésitation vaccinale, à l'échelle mondiale, représente une réelle menace pour la santé publique, comme l'a ainsi classée l'OMS en 2019 parmi les dix menaces pour la santé globale (OMS, 2019). La profession vétérinaire en est également victime, avec des propriétaires qui parfois par conviction refusent la vaccination. D'autres courants de pensée, ou modes de vie ont également une influence sur la médicalisation du chien et du chat donc sur la pratique vétérinaire, comme la lutte contre l'utilisation non raisonnée de molécules chimiques (antibiotiques, antiparasitaires, pesticides, etc.) ou bien des pratiques comme l'alimentation BARF (Biologically Appropriate Raw Food) chez le chien ou le véganisme chez l'Homme.

\section{La vaccination, pilier de la consultation du praticien généraliste}

Appliquée aux chiens et aux chats, la vaccination permet d'établir un état de protection dont le résultat dépend autant de la maladie concernée que du vaccin utilisé. Cette protection peut s'exprimer de différentes façons : soit en supprimant l'infection (stratégie prophylactique idéale qui reste néanmoins conditionnée à la physiopathologie de la maladie et à la nature de l'agent pathogène), soit en diminuant ou supprimant l'expression clinique de la maladie, et/ou l'excrétion de l'agent pathogène. De nombreux vaccins sont disponibles pour les chiens et les chats. Ils ont permis, conjointement à l'utilisation de la vaccination orale du renard, de participer à l'éradication de la rage terrestre en France, maladie zoonotique d'importance majeure, et également de diminuer l'incidence et la prévalence de maladies canines ou félines (maladie de Carré, hépatite infectieuse canine dite de Rubarth, etc.). Les vaccins disponibles ont évolué en gagnant en innocuité. Les protocoles de vaccination, détaillés réglementairement dans les résumés des caractéristiques produit du dossier réglementaire d'autorisation de mise sur le marché ont pu être précisés grâce aux connaissances acquises depuis les années 1960-1970. Ils font l'objet de nombreuses recommandations internationales ou nationales pour accompagner au quotidien les praticiens dans leurs applications (Day, 2016). La vaccination est également un acte fondateur de l'activité du praticien généraliste. Sa pratique fait la part belle à la prise en charge de l'animal en bonne santé. Ces consultations indifféremment dénommées «consultation vaccinale», ou plus récemment «consultation annuelle de santé» ou " bilan de santé ", permettent au vétérinaire de maintenir le lien avec les propriétaires d'animaux en bonne santé. De nombreuses études sont réalisées chaque année pour étudier le comportement des propriétaires avec leur animal de compagnie et permettent de confirmer cette relation privilégiée (Cohen, 2002). L'objectif de l'adoption d'un animal de compagnie, chien ou chat, correspond dans la plupart des cas au désir d'établir une relation affective avec l'animal adopté. Le maintien de conditions permettant une relation optimale pérenne, grâce à un état de santé qui interfère le moins possible avec la qualité de cette relation - incluant les stratégies recommandées en médecine préventive, est un point fondamental à considérer.

\section{Médecine préventive et vaccination : des problé- matiques communes}

L'histoire de la vaccination, si elle représente sans contestation possible la stratégie médicale qui a sauvé le plus de vies depuis sa mise en place (Soubeyran, 2003), montre que son développement n'a pas été simple et linéaire. Bien qu'il s'agisse de médicaments dont l'innocuité est très élevée, il n'en reste pas moins que des effets indésirables existent. Il s'agit donc dans le cas de la vaccination d'une réelle analyse de risques dans laquelle les bénéfices attendus et perçus entrent en compétition directe avec les risques liés à l'acte vaccinal, la perception du risque de la maladie d'autant plus ténue que la maladie est correctement gérée avec une prévalence réduite, l'adéquation de la stratégie vaccinale avec le mode de vie, les croyances et courants de pensée, et l'accès aux informations. La preuve scientifique de l'efficacité et le rapport bénéfices/risques doivent être établis et accessibles pour pouvoir être communiqués de la façon la plus transparente possible par des sources de communication fiables et crédibles par tous ou du moins la majorité. Ces éléments sont clairement en faveur de la lutte contre l'hésitation vaccinale, avec, comme clé, une stratégie de communication précise, définie, et rigoureuse, comme a pu l'expliquer Anne Claire Siegrist lors de la conférence inaugurale du semestre d'automne de l'Université de Genève, le 15 septembre 2015 (Siegrist, 2015). La Médecine Préventive, et particulièrement celle des carnivores domestiques - celle des animaux de production étant généralement réglementée pour des raisons de santé publique, fait face aux mêmes problématiques détaillées ci-dessous.

La nécessité accrue de démonstrations de l'efficacité. L'animal pour lequel une stratégie de prévention est proposée n'est pas malade, du moins dans le cadre de la prévention primaire et secondaire comme cela sera expliqué ultérieurement. Pour certains cas, il est extrêmement compliqué en médecine vétérinaire de mettre en évidence par des études scientifiques l'efficacité d'une stratégie de prévention. La comparaison de lots pour obtenir une démonstration rigoureuse nécessiterait à la fois de maîtriser l'épidémiologie des affections ciblées par une méthode préventive et également de constituer des lots considérables, multicentriques, comme les études en médecine humaine, le font. Certaines données, bien que reposant sur des critères scientifiques, ne peuvent donc pas 
facilement obtenir des preuves scientifiques fondées selon le principe de l'EBM - Evidence Based Medicine. Par exemple, le système immunitaire des chiens et des chats est considéré comme développé à six mois, et il a été décrit une persistance possible des anticorps maternels jusqu'à cinq mois pour certaines maladies, notamment le typhus chez le chat (Jakel, 2012). Pour autant, s'il est recommandé dans les protocoles d'usage de valences vaccinales essentielles, qu'une injection soit effectuée à l'âge de six mois, il est très compliqué d'en apporter une preuve rigoureuse selon les critères EBM à l'échelle de la population canine ou féline.

La balance bénéfices/risques du traitement proposé. Tout traitement médical s'il est actif, présente des effets bénéfiques et des effets indésirables, aussi faibles et maitrisés soient-ils. Les vaccins, les traitements antiparasitaires, et d'une façon générale tous les traitements préventifs doivent d'abord démontrer une innocuité extrêmement élevée pour des raisons évidentes d'acceptation : il n'est pas envisageable de rendre un animal malade alors qu'il est sain au moment de l'application du traitement prophylactique d'une affection hypothétique. Pour autant, ces effets indésirables vont s'exprimer dans la population lors de la généralisation du traitement. S'ils ne sont pas connus par les propriétaires, ou s'ils sont tus par les professionnels, alors ils représentent la meilleure arme pour les opposants à ces stratégies de prévention. Cela souligne une fois de plus la nécessité de la pharmacovigilance, particulièrement dans le domaine des vaccins (Montastruc, 2016).

Une communication claire, transparente, personnalisée. Le professionnel de santé se doit donc à la fois d'expliquer les risques encourus par l'animal, les propositions de gestion de ces risques, les bénéfices et risques attendus pour permettre au propriétaire de s'engager dans la prévention proposée. Cette communication nécessite à la fois des compétences propres, du temps de consultation, et de la patience pour permettre au propriétaire d'apporter son consentement éclairé à la stratégie préventive. Cette démarche repose sur le procédé d'analyse de risques détaillé ultérieurement.

\section{LA MÉDECINE PRÉVENTIVE, UNE DÉMARCHE MÉDICALE RIGOUREUSE}

\section{De la prévention en médecine humaine à la méde- cine préventive du chien et du chat}

À partir de la constitution de l'OMS de 1948 a été ultérieurement définie la prévention comme étant " l'ensemble des mesures visant à éviter ou réduire le nombre et la gravité des maladies, des accidents et des handicaps ". Trois stades de prévention ont été précisés :

- la prévention primaire dont l'objectif est de diminuer l'incidence de la maladie ;

- la prévention secondaire qui agit pour diminuer la durée de la maladie et ainsi diminuer sa prévalence ;
- la prévention tertiaire dont l'objectif est de diminuer les conséquences liées à la maladie ainsi que la récidive. Un quatrième stade de prévention a été ajouté ultérieurement, la prévention quaternaire, qui vise à diminuer les effets iatrogènes et à accompagner la fin de vie (figure 1). À partir de cette définition de l'OMS, la médecine préventive du chien et du chat a été considérée par les enseignants français des quatre Écoles vétérinaires de la discipline, en 2016, comme étant une discipline clinique des sciences vétérinaires qui s'attache tout au long de la vie de l'animal, à préserver son état de bonne santé, à identifier et limiter les risques de sa dégradation, ainsi qu'à empêcher la transmission de zoonoses (Freyburger et al. 2016a). Lors de ces consultations, la vaccination, la lutte contre les parasites externes et internes, la nutrition, la stérilisation ou la mise en reproduction, le développement, l'hygiène, le dépistage clinique et génétique d'anomalies héréditaires, et la mise en conformité avec la réglementation en vigueur sont autant de sujets non exhaustifs qui doivent être abordés, afin de transmettre au propriétaire une information capitale pour le maintien le plus longtemps possible de la relation propriétaire-animal, telle qu'elle a été souhaitée à l'acquisition de l'animal de compagnie.

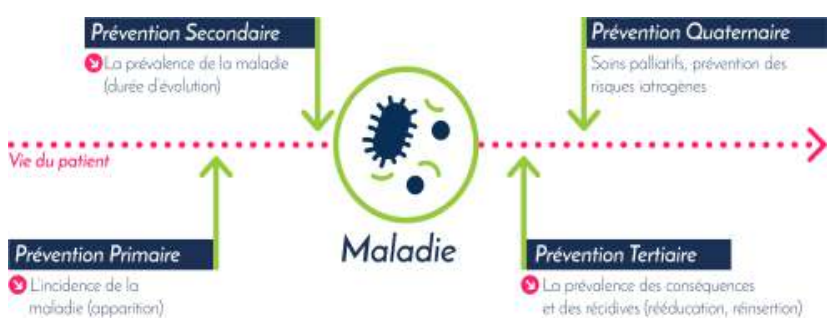

Figure 1 : les quatre étapes de la prévention

L'objet de la médecine préventive est de déterminer quelles sont les stratégies à mettre en œuvre pour permettre à l'animal de tirer le meilleur profit de cette dernière. L'autre aspect important de la médecine préventive est la prise en charge tout au long de la vie de l'animal et non plus en rapport avec un épisode temporel représenté par la maladie. Pour le propriétaire, cela modifie considérablement la relation avec la clinique vétérinaire, et pour le vétérinaire, cela donne du sens à la construction d'un plan de soins et complémentairement à la prise en charge ponctuelle d'une maladie ou d'un acte de prévention dépourvu de lien avec les autres événements de la vie de l'animal. Il est donc nécessaire d'appréhender cette approche spécifique de la santé du chien et du chat avec une méthodologie rigoureuse et systématique.

\section{La démarche par analyse de risques, une métho-} dologie rigoureuse et systématique

Quatre étapes sont nécessaires à l'application d'une analyse de risques telle qu'elle a été définie notamment dans le code zoo-sanitaire international de l'OIE (Zepeda Sein, 2002 ; Dufour, 2011 ; OIE, 2019 ; Freyburger, 2021). 
Identification $\boldsymbol{d} \boldsymbol{u}$ danger. Le danger représente tout ce qui est susceptible de causer un dommage pour la santé de l'animal et celle du propriétaire dans le cas par exemple de zoonoses. Cela peut être un agent pathogène (ex : un parvovirus félin), une situation (ex : l'accès à une alimentation en quantité et qualité inappropriée), un événement (vacances, déménagement, grossesse/naissance, etc.), une absence de moyens (choix de l'euthanasie par manque de moyen pour payer les traitements adéquats), etc.

Appréciation des risques. La probabilité de la survenue de l'exposition au danger et la gravité du danger permettent d'apprécier la probabilité d'apparition d'un dommage, encore appelé risque. La probabilité de survenue dépend de la fréquence du danger et de l'importance de l'exposition de l'animal à ce danger. Plusieurs stratégies permettent d'agir sur un risque : soit en diminuant ou supprimant le danger, soit en diminuant ou supprimant l'exposition de l'animal au danger, soit en diminuant ou supprimant la gravité du dommage. L'évaluation de la gravité du dommage peut être limitée à l'individu ou inclure les conséquences pour le propriétaire (zoonose) ou pour les autres animaux du foyer (maladie contagieuse). Les commémoratifs, les tests diagnostiques et de dépistage permettent de préciser l'appréciation du risque. Cette appréciation des risques passe donc par une estimation de la probabilité de survenue et une évaluation des conséquences (comparaison entre l'estimation et un seuil prédéfini de risque acceptable) puis par une hiérarchisation des risques par rapport aux besoins de l'animal et aux attentes du propriétaire.

Gestion $d u$ risque. Le propriétaire décide s'il accepte la prise de risque ou non. Dans ce dernier cas, le praticien liste les mesures de gestion existantes et les hiérarchise en fonction de leurs conséquences sur le risque, leur coût et leur faisabilité. Il détermine les bénéfices et les limites et estime les risques résiduels réduits qui découlent des limites des décisions prises précédemment.

Communication. Le praticien informe les propriétaires et les implique dans la mise en place des stratégies décidées en commun accord pour en améliorer l'efficacité et l'observance. Le vétérinaire privilégiera les stratégies de suppression des risques ou proposera le cas échéant, de réduire les risques avec les bénéfices attendus et les limites potentielles. Qu'il s'agisse par exemple de l'évaluation des risques parasitaires, ou de l'évaluation des risques nutritionnels, la connaissance des besoins physiologiques de l'animal, de son mode de vie, du mode de vie de ses propriétaires sont primordiaux dans la stratégie proposée. Faut-il systématiquement et indifféremment traiter les chiens et les chats contre les parasites externes ou les parasites internes avec une molécule à large spectre? A l'inverse, est-ce que les traitements antiparasitaires recommandés couramment sont réellement adaptés aux risques encourus par l'animal, lorsqu'il change d'environnement (vacances, déménagement, nouveaux congénères ou personnes à risques dans l'entourage) ? Faut-il promouvoir la stérilisation de l'animal dont l'état d'embonpoint est excessif en regard de l'impact sur la prévention des tumeurs mammaires ? Comment et quand mettre en place une stratégie de maintien de l'hygiène bucco-dentaire pour éviter la dégradation de la cavité buccale par la présence de tartre? Cette liste non exhaustive d'exemples permet de comprendre que la démarche de médecine préventive ne peut être mise en place qu'avec l'accumulation de connaissances permettant de comprendre la physiopathologie de l'affection étudiée, puis les mécanismes et facteurs permettant d'agir selon les besoins au niveau préventif primaire, secondaire, tertiaire voire quaternaire pour limiter les risques d'apparition de la maladie concernée. Contrairement à la vaccination, stratégie de prévention primaire par excellence, qui s'est historiquement développée comme tel, par nécessité, les stratégies médicales de prévention représentent souvent un stade très avancé de connaissance et de compréhension des mécanismes physiopathologiques et thérapeutiques des maladies ou affections que le praticien peut envisager de prévenir. Ainsi, pour prévenir la récidive en cas de calculs vésicaux chez le chat, il a été nécessaire de découvrir cette affection de l'appareil urinaire du chat, d'être capable de la traiter (chirurgie, prise en charge médicale), puis d'analyser les calculs pour en déterminer la composition, de comprendre leur mode de formation, et enfin, d'avoir les connaissances en nutrition animale pour éviter que ces calculs ne se forment à nouveau. La prévention, tertiaire dans ce cas, n'intervient que tardivement dans l'histoire de l'affection, et nécessite la maîtrise des connaissances des différentes étapes physiopathologiques.

\section{UNE ÉQUIPE TRIPARTITE AU SERVICE DE LA SANTÉ DE L'ANIMAL POUR PRÉSERVER LE LIEN HOMME-ANIMAL}

La médecine préventive repose donc sur une analyse de risques qui doit être expliquée pour être comprise et acceptée, grâce à une méthode de communication adaptée (Silverman, 2013). Un réel lien se crée entre les propriétaires et l'équipe soignante, composée des vétérinaires et des auxiliaires spécialisés vétérinaires (ASV)

\section{Des propriétaires engagés qui deviennent acteurs}

Les propriétaires d'animaux sont réellement engagés et prêts à s'investir dans les démarches de prévention. En 2014, dans une enquête menée dans un panel de 2107 personnes, composé de 442 vétérinaires, 234 ASV et 1431 propriétaires de chiens (881) et de chats (540) concernant la médecine préventive, il ressort que les propriétaires sont prêts à s'investir fortement, y compris financièrement, pour maintenir leur animal en bonne santé, à partir du moment où ils ont compris les recommandations formulées par le vétérinaire (Freyburger, 2015, comm. pers.). Cette compréhension passe par une explication claire et exhaustive des besoins de l'animal, et des stratégies de prévention applicables, donc de la démarche mise en place en consultation. Prenons l'exemple d'une analyse de risques parasitaires : les habitudes et lieux de vie du propriétaire et de l'animal doivent impérativement être évalués, et pris en compte pour adapter le 
traitement au risque réellement encouru par l'animal et par le propriétaire. Cela nécessite de poser entre autres, des questions sur les vacances, les déplacements en dehors du lieu d'habitation, l'état de santé ou de fragilité des personnes et autres animaux qui entourent l'animal pour adapter au mieux les cibles du traitement, les molécules à utiliser, les modes d'administration et la posologie.

Ce traitement devrait être systématiquement expliqué avec les bénéfices et les limites que le propriétaire peut en attendre. Un traitement antiparasitaire externe, par exemple, n'empêchera pas les puces d'être présentes sur le chien. Il permettra de les tuer, par différents mécanismes et plus ou moins rapidement en fonction des molécules utilisées. Autre exemple, un chat vacciné contre le " coryza ", sera protégé contre le coryza pour le propriétaire. Or l'acception du terme protégé ne sera pas la même pour le propriétaire qui comprend "mon chat ne sera pas malade", et pour le vétérinaire, qui sait que le chat présentera une forme symptomatique atténuée à la suite d'une contamination par les agents pathogènes contre lesquels le chat aura été vacciné. En l'absence de compréhension des choix effectués, le propriétaire ne respectera pas la prescription, ou bien ira chercher ailleurs des informations complémentaires plus ou moins fiables. La communication mise en place par l'équipe soignante est donc fondamentale dans cette approche pour permettre son application.

\section{Des vétérinaires qui évoluent du statut de soignant à celui de partenaire de santé}

Une différence fondamentale entre l'exercice professionnel de la pathologie médicale et chirurgicale, et celui de la médecine préventive réside principalement dans le sujet de l'étude. L'animal suspect d'être malade dans le premier cas, est présumé sain dans le second. L'approche clinique dans les deux cas est similaire : une méthode d'analyse diagnostique ou de risques, passant par une anamnèse, un examen clinique et la proposition de stratégie thérapeutique ou prophylactique, pourtant la communication et l'approche de la relation avec le client sont radicalement opposées.

L'objectif du clinicien face à un animal symptomatique est d'établir par des méthodes rigoureuses, idéalement un diagnostic dit " de certitude ". Ensuite, en fonction des connaissances médicales et scientifiques actuelles, le praticien proposera les solutions les plus adaptées à la prise en charge de l'animal en considérant les volontés, les choix et les moyens du propriétaire pour résoudre le problème. Dans cette situation, le rôle du vétérinaire est évident et incontournable. Si le propriétaire peut surseoir à la consultation, il se rendra tôt ou tard avec son animal dans une structure vétérinaire pour une prise en charge de son animal par les personnes de l'art. Le praticien est un acteur irremplaçable de la situation. Le propriétaire suivra les recommandations du praticien, détenteur à la fois du savoir et de la compétence permettant l'amélioration de l'état de santé de son animal. Ainsi, le praticien ordonnera des traitements, qui seront acceptés par le propriétaire, impuissant par lui-même sur la situation. De plus, les actes médicaux et chirurgicaux du vétérinaire seront effectués dans le cadre de l'exercice réglementé de la profession vétérinaire.
Dans une situation de médecine préventive, l'animal présumé sain, n'a pas de nécessité d'être amené en consultation auprès du vétérinaire. Cette démarche, accompagnée par les professionnels de santé (vétérinaires et ASV) grâce à des outils de communication les plus appropriés possibles, est et reste volontaire de la part du propriétaire. Par ailleurs, l'ensemble des stratégies prophylactiques proposées auront pour objectif principal de maintenir l'animal en bonne santé.

Si les conséquences sont réelles, la perception du résultat sera nulle pour le propriétaire. Il va en effet devoir prendre en compte, parfois quotidiennement, des recommandations modifiant ses habitudes, administrer des traitements et dépenser de l'argent, pour un bénéfice invisible, puisque l'animal restera dans la situation dans laquelle il est : en bonne santé. Seuls l'échec ou le dysfonctionnement pourront éventuellement, aboutir à une déception de la part du propriétaire, au regard de l'engagement fourni. La relation de communication entre le propriétaire et le vétérinaire est alors rééquilibrée, le premier n'ayant pas nécessairement besoin du deuxième : d'une part parce que la situation n'exige pas à priori l'intervention d'un professionnel de santé animale pour rétablir une situation physiologique qui serait défavorablement modifiée, d'autre part, parce que les effets ne seront pas "visibles ", pour une bonne partie des stratégies prophylactiques proposées.

De la position de soignant dans la première situation, le praticien devient accompagnateur de la prise en charge de la santé de l'animal tout au long de sa vie. D'obligatoire et contrainte, la consultation devient volontaire. La relation de communication entre le professionnel et son client évolue donc : de l'ordonnance, elle se transforme en partenariat avec une plus grande implication du client dans la prise de décision.

\section{Les auxiliaires spécialisés vétérinaires, pierre angulaire de l'équipe}

Les ASV ont un rôle crucial dans la médicalisation des animaux. Ils sont à la fois en contact avec le propriétaire avant et après la consultation et souvent entre deux consultations - à l'occasion d'un passage à la clinique ou d'un appel téléphonique. À la fois par rapport aux rendez-vous pris pour des consultations et actes de prévention, et également pour la bonne explication des recommandations et prescriptions des vétérinaires, les ASV doivent pouvoir faire preuve de compétences techniques importantes, et de compétences de communication indispensables. Souvent, leur implication permettra d'accroître l'observance et le suivi des animaux. Les ASV pourront transmettre des informations capitales aux vétérinaires pour l'adaptation des stratégies prophylactiques et des traitements. Ils et elles pourront également être impliqué(e)s dans l'explication des gestes d'hygiène, ou d'application correcte des traitements. Tous les membres de l'équipe médicale, vétérinaires et ASV, sont donc fortement concernés par la bonne mise en place de la médecine préventive (Figure 2). Chacun, avec ses compétences, accompagne les propriétaires dans la bonne mise en place des stratégies visant à maintenir l'animal en bonne santé. Compte tenu de l'aspect volontaire de cette pratique, et du fait que la réussite des traitements envisagés dépend pour partie des 
propriétaires, un réel partenariat médical doit être construit, non pas en imposant un ensemble de soins, mais bien en proposant toutes les possibilités nécessaires à la santé de l'animal et en accompagnant le propriétaire dans la prise de décision adéquate.

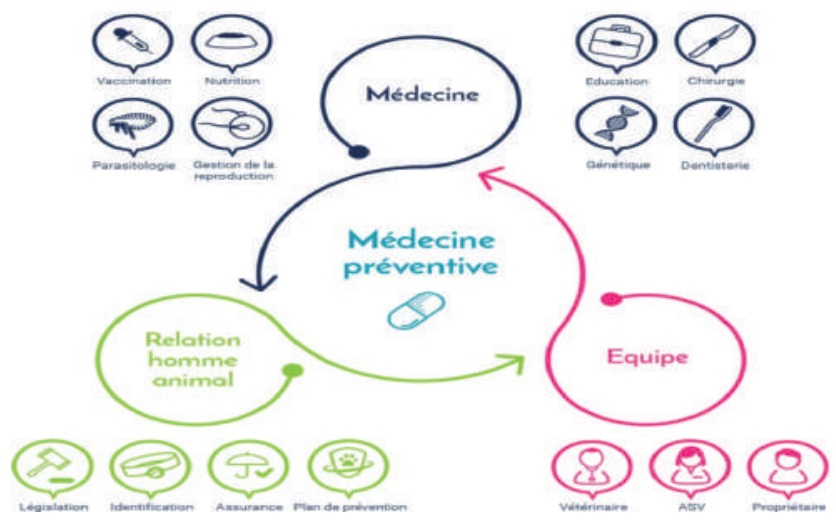

Figure 2 : Rôle du partenariat entre l'équipe médicale et le propriétaire permettant l'application de stratégies de médecine préventive au service de la préservation du lien homme-animal

Une prise de conscience s'impose donc pour mettre en avant une démarche qui paraît chronophage et qui pourtant doit être systématique et rigoureuse pour être correctement comprise par le propriétaire. Actuellement, la consultation de vaccination ou le bilan annuel de santé - deux dénominations réductrices sont trop souvent encore des variables d'ajustement de l'emploi du temps. Or, les besoins des animaux consultés ne varient pas en fonction de la charge de travail de la clinique ou de l'heure de passage en consultation. Par ailleurs, l'acceptation de la démarche par le propriétaire va de pair avec la transparence et la compréhension de ces bienfaits et limites : l'explication dès le premier contact avec l'animal sur les grandes étapes à envisager tout au long de sa vie, et sur ce qu'il sera envisageable de mettre en place, facilitera l'acceptation ultérieure par le propriétaire (Freyburger, 2016b).

\section{LA MÉDECINE PRÉVENTIVE, UNE DISCIPLINE EN PLEINE STRUCTURATION}

Les Écoles vétérinaires ont historiquement dispensé un enseignement disciplinaire, la consultation de vaccination étant alors traitées dans le cadre des maladies réglementées (actuellement unité d'enseignement de "pathologie infectieuse " ou de «maladies contagieuses »), compte tenu du caractère réglementaire de la vaccination antirabique. Les services de vaccination des quatre Écoles ont désormais évolué en services cliniques de médecine préventive, dépendant de l'hôpital des petits animaux, et dans lesquels une démarche transversale de médecine préventive est appliquée. Chacune des Écoles est pourvue d'un service, au sein duquel le processus de prise en charge de l'animal sain dans son intégralité et tout au long de sa vie est enseigné. Un enseignement théorique a lieu par ailleurs, identifié pour les étudiants comme un enseignement de médecine préventive, depuis 2019 à VetAgro Sup (Marcy l'Etoile - 69). Des passerelles se créent progressivement en enseignement : passerelles intradisciplinaires avec les enseignants de médecine préventive qui travaillent de concert et structurent conjointement l'évolution pédagogique de la discipline. Des passerelles interdisciplinaires également avec les enseignants de pathologie médicale des animaux de compagnie incluant les équidés. Toutes œuvrent collectivement à un développement des connaissances permettant d'améliorer la précocité de prise en charge de la santé animale en médecine préventive. Depuis 2013, une réunion annuelle des enseignants de médecine préventive des quatre Écoles françaises se tient conjointement à celle des enseignants de pathologie médicale permettant de travailler sur des thématiques communes. La profession, par le biais de l'Association française des vétérinaires pour animaux de compagnie (AFVAC) a créé en mars 2019 un groupe d'étude en médecine préventive (GEMP), dont l'objectif est de participer tant au développement des connaissances et compétences dans le domaine de la médecine préventive que de promouvoir la discipline et son application dans la profession vétérinaire par le biais de formation continue. Ces démarches qui visent à accroître les connaissances, l'effort de structuration dans la discipline afin de permettre sans nul doute une évolution générationnelle du quotidien des vétérinaires praticiens.

\section{CONCLUSION}

La médecine préventive, plus que les autres approches médicales, entre dans le quotidien du propriétaire, avec pour principal objectif de permettre de maintenir le lien avec son animal de compagnie dans les meilleures conditions possibles. Cette démarche " invisible ", ne peut réussir qu'à la condition d'un engagement en ce sens, fort, volontaire et conscient de la part du propriétaire, et également de l'équipe médicale. Le propriétaire aura ainsi compris les bénéfices et limites des stratégies proposées par l'équipe soignante. Vétérinaires et ASV mettront à profit en totale synergie leurs connaissances communes de l'animal pour améliorer son bien-être et sa santé. Il s'agit donc d'un réel partenariat plaçant en son centre la santé de l'animal, avec des relations renforcées entre vétérinaires, ASV et propriétaires. Les compétences en communication de l'ensemble de l'équipe permettront la bonne mise en place des approches préventives. Cette discipline en pleine structuration devra par ailleurs bénéficier du développement des connaissances scientifiques dans les domaines de la prévention et de la prédiction, conjointement au développement de celles liées à la pathologie médicale et chirurgicale pour permettre de proposer de nouvelles approches aux propriétaires, au bénéfice de la santé de leur animal. Pour être correctement structurée, une action commune et forte en formation initiale puis tout au long de la vie professionnelle devra également être menée, avec notamment la création de postes statutaires d'enseignants chercheurs en médecine préventive. Ainsi, la médecine préventive qui découle d'un des fondements de la profession vétérinaire représenté par la lutte contre les maladies infectieuses, est désormais une discipline clinique à part entière. Son développement permet, par une démarche scientifique et rigoureuse d'analyse de risques, de maintenir l'animal en bonne santé le plus longtemps possible et de participer à son bien-être. Elle maintient par conséquent et développe le rôle sociétal que l'animal de compagnie occupe auprès des propriétaires des quelques vingt millions de chats et de chiens en France. 


\section{REMERCIEMENTS}

L'auteur tient à remercier l'ensemble des enseignants chercheurs et consultants impliqués en médecine préventive dans les Écoles vétérinaires françaises pour leur engagement dans sa structuration et son développement.

\section{BIBLIOGRAPHIE}

- Bazin H. Histoire des vaccinations. Arcueil : John Libbey Eurotext, 2008.

- Cohen SP. Can pets function as family members? Western Journal of Nursing Research. 2002; 24: 621-638.

- Couteille-Brillet P, Des Garets V, Maubisson L, Rivière A. La confiance envers la vaccination : conceptualisation et influence de différentes sources d'information. Journal de Gestion et d'Économie de la Santé. 2019 ; 37 : 486-509. https://doi.org/10.3917/jges. 196.0486.

- Day MJ, Horzinek MC, Schultz RD, Squires RA. WSAVA Guidelines for the vaccination of dogs and cats. J Small Anim Pract. 2016; 57(1):4-8. doi: 10.1111/jsap.12431.

- Dufour B, Plee L, Moutou F, Boisseleau D, Chartier C, Durand B, et al. A qualitative risk assessment methodology for scientific expert panels. Rev. sci. tech. Off. int. Epiz., 2011; 30(3), 9.

- Freyburger L, Pépin M, Ruvoen N, Le Poder S, Picavet DP, Bisson C et al. La médecine préventive du chien et du chat. Pratique Vét. 2016a ; 51 : 542-552.

- Freyburger L. Planification des consultations de médecine préventive. Pratique Vét. 2016b ; 51, 17-21.

- Freyburger L. La consultation en médecine préventive. La Dépêche Technique. 2021 ; 183, 25-27.

- Genetet B. Que sais-je ? Histoire de l'immunologie. Presses Universitaires de France, 2000.

- Jakel V, Cussler K, Hanschmann KM, Truyen U, König M, Kamphuis E, et al. Vaccination against Feline Panleukopenia: implications from a field study in kittens. BMC Veterinary Research. 2012; 8(62), 1-8. doi:10.1186/17466148-8-62

- Larson HJ, De Figueiredo A, Xiahong
Z, Schulz WS, Verger P, Johnston IG, Jones NS. The state of vaccine confidence 2016: global insights through a 67-country survey. EBioMedicine, 2016, 12, 295-301. doi:10.1016/j.ebiom.2016.08.042.

- Légifrance. Loi $\mathrm{n}^{\circ}$ 2015-177 du 16 février 2015 relative à la modernisation et à la simplification du droit et des procédures dans les domaines de la justice et des affaires intérieures. Disponible à https://www.legifrance.gouv.fr/eli/loi/2015/2/16/2015. 177/jo/article 2. (Consulté le 01/04/2021).

- Légifrance. Proposition de loi no 3661 visant à renforcer la lutte contre la maltraitance animale (2020, 12 14). Disponible à https://www.assemblee-nationale.fr/dyn/15/textes/ 115b3661 proposition-loi (Consulté le 01/04/2021).

- Montastruc JL, Durrieu G, Lacroix I, Lapeyre-Mestre M, Damase-Michel C, Bagheri H, et al. Quelle pharmacovigilance pour les vaccins? Bulletin de l'Académie Nationale de Médecine. 2016 ; 200(2), 241-250.

- Moulin AM. L'Aventure de la vaccination. Paris : Éditions Fayard ; 2016.

- OIE. (2019). Code sanitaire pour les animaux terrestres. Disponible à sur https://www.oie.int/index. php?id=169\& $L=1 \&$ htmfile=chapitre import risk analysis.htm. (Consulté le 01/04/2021).

- OMS. (2019). Ten Threats to global health in 2019. Disponible à https:// www.who.int/news-room/feature-stori es/ten-threats-to-gl obal-health-in-2019. (Consulté le 02/04/2021).

- Pasteur L. Euvres réunies par Joseph Louis Pasteur Vallery-Radot. Paris : Éditions Masson ; 1933.
- Raude J. L'hésitation vaccinale : une perspective psychosociologique. Bulletin de l'Académie Nationale de Médecine. 2016 ; 200(2), 199-209.

- OIE Résolution n¹8 : Déclaration de l'éradication mondiale de la peste bovine et mise en ouvre des mesures de suivi visant à maintenir l'absence de cette maladie dans le monde. (2011). $79^{\text {ème }}$ session générale de l'OIE, (pp. 26-29). Paris. Disponible à https://www.oie.int/fileadmin/ Home/fr/Publications \%20\%26 D ocumentation/docs/pdf/bulletin ¿Bull 2011-4-FRA.pdf (Consulté le 01/04/2021).

- Siegrist AC. Avec ou sans vaccin, un choix de société. Genève, Suisse. 2015. Disponible sur https://mediaserver. unige.ch/play/VN4-15ca-2015-2016 (Consulté le 01/04/2021).

- Soubeyran B. Tolérance des vaccins : faits et spéculations. Innocuité des vaccins : faits et spéculations. Médecine et Maladies Infectieuses. 2003 ; 33(6), 287-299. https://doi.org /10.1016/S0399-077X(03)00181-1.

- Silverman J, Kurtz S, Draper J. Skills for communicating with patients. 2013 ( $3^{\text {rd }}$ Ed). Radcliffe Publishing.

- VetFuturs France. Le Livre Bleu. comprendre et anticiper les mutations. 2018. Disponible sur https://www.v eterinaire.fr/fileadmin/user upload/ documents/actualites/vetfuturs/ livre bleu VF versionBDef 0407 2018.pdf (Consulté le 01/04/2021).

- Zepeda Sein C. L'analyse de risque : un outil d'aide à la décision pour la prophylaxie et la prévention des maladies animales. (2002) 257-261. Disponible sur https://www.oie.int/ doc/ged/D2940.PDF (Consulté le 01/04/2021). 